\title{
El género Heliconius Kluk, 1708 en dos habitats de diferente grado de conservación en la Amazonia colombiana y aportes para su conservación
}

\author{
Nicol Rueda-M ${ }^{1, *}$, M. Gonzalo Andrade-C. ${ }^{2}$ \\ ${ }^{1}$ Facultad de Ciencias, Departamento de Biología, Universidad Nacional de Colombia, Bogotá, Colombia \\ ${ }^{2}$ Facultad de Ciencias, Instituto de Ciencias Naturales, Universidad Nacional de Colombia, Bogotá, Colombia
}

\begin{abstract}
Resumen
El objetivo del presente estudio fue determinar las especies del género Heliconius presentes en hábitats con diferente grado de conservación en la Amazonia colombiana, y así responder a la pregunta de si ¿el grado de conservación de los bosques en el Amazonas colombiano afectan la diversidad del género Heliconius? esto mediante la recolección de este género a lo largo de seis meses consecutivos en dos sitios de diferente grado de conservación. La comunidad del género Heliconius en esta zona está compuesta por 11 especies, de las cuales 9 fueron recolectadas en Leticia (sitio1 - menor grado de conservación) y 8 en Puerto Nariño (sitio2 - mayor grado de conservación). En el sitio2 no se encontraron dos subespecies de la especie $H$. numata ni la especie $H$. hecale humboldti. Si bien los sitios son significativamente diferentes en su estado de conservación, se puede decir que el sitiol presenta una afectación del bosque intermedia, por tanto, se considera que los resultados de este trabajo en cuanto a un mayor número de individuos en el sitio más conservado, es una primera muestra de la afectación del grado de conservación del bosque en las poblaciones de mariposas y que el resultado de una diversidad mayor en el sitio1, se puede explicar en parte por la dificultad de captura en el sitio 2 y por otra parte se debe considerar que este género se le ha visto en otros estudios ser más resistentes a la perturbación gracias a la plasticidad ecológica que presentan. C 2016. Acad. Colomb. Cienc. Ex. Fis. Nat.
\end{abstract}

Palabras clave: Heliconius; Amazonas; Conservación; Diversidad.

The Heliconius genus Kluk, 1708 in two different habitats degree of conservation in the Colombian Amazon and contributions to conservation

\begin{abstract}
The objective of this study is to determine the species of the genus Heliconius in habitats with different degrees of conservation in the Colombian Amazon, and thus answer the question of whether does the degree of conservation of forests in the Colombian Amazon affect gender diversity Heliconius? this by collecting this genus over six consecutive months in two different sites degree of conservation. Genus Heliconius community in this area is composed of 11 species, of which 9 were collected in Leticia (Site1 - Low Degree of Conservation). And 8 in Puerto Nariño (Site2 - High Degree of Conservation). In Site 2 we did not find two subspecies of the species H. numata nor the species $H$. hecale humboldti. Although sites are significantly different in their conservation status, it can be said that the Site 1 has an affectation of intermediate forest, therefore, it is considered that the results of this work in terms of a greater number of individuals on the Site best preserved, is a first sign of involvement on the degree of forest conservation in butterfly populations and that the result of greater diversity in Site 1, can be explained in part by the difficulty of capturing the Site 2 and moreover should be considered that this genus has been seen in other studies to be more resistant to disturbance by presenting the ecological plasticity. C 2016. Acad. Colomb. Cienc. Ex. Fis. Nat.
\end{abstract}

Key words: Heliconius; Amazonas; Conservation; diversity.

\section{Introducción}

Son muchos los estudios que de este grupo se han hecho alrededor del mundo y se dice que es uno de los grupos mejor estudiados dentro de los artrópodos y que dentro del grupo de mariposas, el género Heliconius, es el más estudiado, ya que de él se posee buena información sobre su sistemática, comportamiento, biología de sus inmaduros, relaciones plantas hospedera, coevolución, mimetismo, genética y algunos trabajos de ecología poblacional (Turner 1971, Ehrlich, P. \& Gilbert, L., 1973, Cook, L., Thomason, E., \& Young,

\footnotetext{
*Correspondencia:

Nicol Rueda-M, nmruedam@gmail.com

Recibido: 02 de junio de 2016

Aceptado: 25 de noviembre de 2016
} 
A., 1976, Araujo, A.M., 1980, Mallet, J.L.B. \& D.A. Jackson, 1980, Brown, K., 1981, Ehrlich, P., 1984, Mallet, J., 1986, Quintero, H. E., 1988, Mallet, J. \& L. E. Gilbert., 1995, Jiggins, C.D., et al., 2001). Sin embargo, no son tan abundantes los trabajos en el departamento del Amazonas en Colombia, ya que, dentro de los antecedentes revisados se encontró que de las especies del género Heliconius en esta zona del país se tienen reportes aislados de la presencia de especies y subespecies del género pero no se encontraron trabajos a largo término ni comparativos de presencia de especies en bosques con diferente grado de conservación.

Se hizo así pertinente llevar a cabo esta investigación buscando aportar al conocimiento de las mariposas, el cual comienza a verse afectado por las diferentes problemáticas ambientales, tanto así que a nivel mundial 724 son las mariposas que se encuentran registradas en la Lista Roja de IUCN (IUCN, 2016). En esta lista hay actualmente 10 especies de origen colombiano; lo cual nos indica que hace falta mucha más investigación sobre este grupo que no solo aportaría a su conservación sino también a muchos otros seres vivos al ser estas reconocidas como un grupo indicador ecológico valioso, por su abundancia, facilidad de encuentro y manejo en campo, por su estabilidad espacio temporal y por qué se trata de un grupo taxonómicamente bien estudiado, así como, por ser sensibles a perturbaciones mínimas y presentar fidelidad ecológica (Andrade-C., M.G., 1998). Por estas razones, todos los estudios que se lleven a cabo con mariposas aportan no sólo a su conocimiento sino también al del hábitat en donde se encuentren y acerca de otros seres vivos con los que ellas tienen relaciones.

De esta manera, la presente investigación buscó aportar información sobre las especies del género Heliconius presentes en el Amazonas y las diferencias, si existen, entre las comunidades de mariposas de este género en cuanto a la presencia de sus especies en dos hábitats de diferente grado de conservación y así poder aportar a la conservación de las mariposas del Amazonas y en particular del género Heliconius que aunque es uno de los grupos taxonómicos mejor estudiados, no son la excepción a la falta de información tanto biológica como de dinámica de sus poblaciones. Es así como, este trabajo tuvo por objetivo determinar las especies del género Heliconius presentes en hábitats con diferente grado de conservación en los bosques lluviosos del sur del Amazonas colombiano, y así responder a la pregunta de si ¿el grado de conservación de los bosques en el Amazonas colombiano afectan la diversidad del género Heliconius? esto mediante la recolección de los individuos de este género a lo largo de seis meses consecutivos en dos hábitats de diferente grado de conservación. Al final se logró obtener la lista de las especies del género Heliconius que habitan en esta zona, una comparación de las especies entre los sitios y a partir de un análisis de los resultados se estableció una propuesta que contiene medidas de gestión, mejora y conservación de los hábitats en cuestión en función a este grupo de mariposas.
Este trabajo toma importancia si se tiene en cuenta que el género Heliconius presenta un amplio polimorfismo intraespecífico en los caracteres de los patrones de color del ala principalmente a lo largo del Rio Amazonas, esto hace que en esta zona (Amazonas - colombiano) sea común encontrar una gran variedad de subespecies, resultante de la hibridación y otros procesos evolutivos, por lo que esta zona ha sido denominada como de contacto o sutura interracial. Así mismo, se considera que la mayoría de las subespecies de tierras bajas no representan especies incipientes, dado que hay una alta tasa de flujo de genes lo que conlleva a la no divergencia entre polimorfos locales y a la no diferenciación a nivel molecular, de esta manera, se considera que estas subespecies son relativamente jóvenes en relación a las subespecies distribuidos en el área Andina y que si bien se encuentra en un proceso de especiación parapátrica, esta es muy lenta debido a que el Amazonas presenta menos barreras de aislamiento debido a su menor diferencia en el hábitat (Rosser, N., et al., 2012), concluyendo de esta manera que las subespecies de una misma especie en esta zona pertenecen a un solo taxón.

Por lo tanto, en este artículo se presentan las especies encontradas, así como, las subespecies o polimorfos de cada una de estas, y se aclara que el análisis de la cantidad de especies recolectados de las subespecies $H$. erato reductimacula y $H$. erato lativitta; $H$. melpomene malleti y $H$. melpomene vicina y $H$. numata aurora, $H$. numata silvana y $H$. numata arcuella son tomadas como un solo taxón denominados en este trabajo como H. erato spp?, H. melpomene spp? y H. numata spp? respectivamente, esto debido a lo mencionado en el párrafo anterior así como a la verificación que hizo en campo de la presencia de flujo de genes entras estas subespecies. Este nombre es asignado de esta manera, debido a que se decide no dar un nombre taxonómico definido a cada uno de estos grupos de subespecies de mariposas siendo consciente de que esto implicaría hacer un análisis fuera de los objetivos de este trabajo, que incluirían entre otras cosas, analizar el patrón de alas, las genitalias y los resultados moleculares, lo que permitiría decir si estos polimorfos pertenecen todos a una subespecie ya descrita dentro de $H$. numata; o a la descripción de una nueva subespecie o al de una nueva especie dentro del género Heliconius.

\section{Materiales y métodos}

El muestreo fue llevado a cabo en dos áreas del sur del Departamento de Amazonas de Colombia. Las áreas de estudio fueron "sitiol" (S0408'0.50"- W69 56'29.50"), en el municipio de Leticia y "sitio2" (S 03 46'12.3" - W

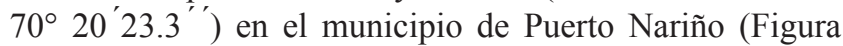
1). De acuerdo a la clasificación climática de KöppenGeiger las áreas de muestreo son del tipo Af - Ecuatorial, esta zona de manera general se caracteriza por presentar lluvias repartidas a lo largo del año por lo que no hay una estación seca, ya que todos los meses superan los $60 \mathrm{~mm}$, las 

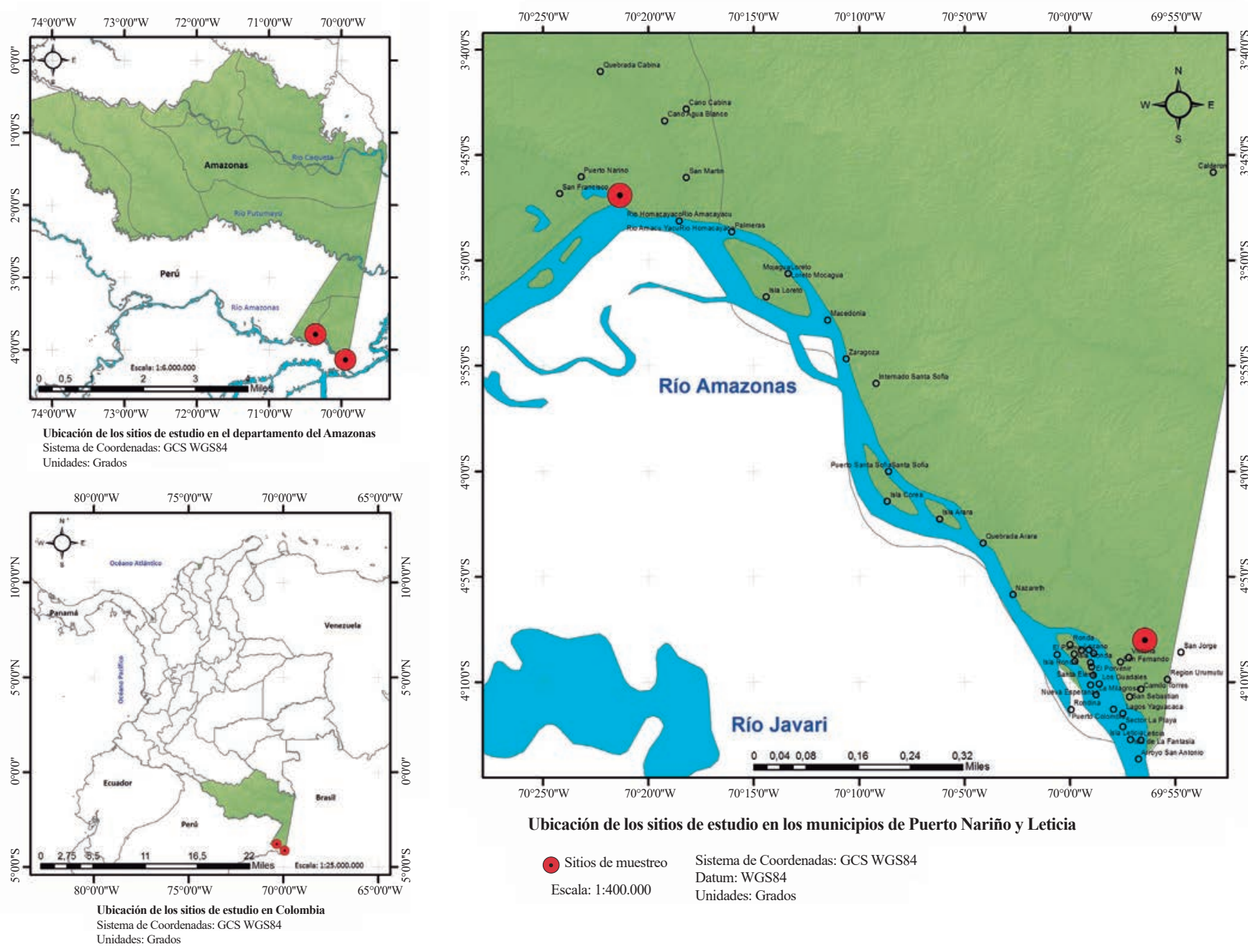

Ubicación de los sitios de estudio en los municipios de Puerto Nariño y Leticia

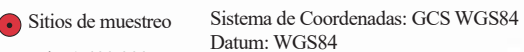

Escala: 1:400.000 Datum: WGS84

Figura 1. Departamento del Amazonas - Colombia y sitios de muestreo.

temperaturas son altas, las oscilaciones diarias mayores que las oscilaciones anuales, la radiación solar es muy intensa y en el ambiente está siempre saturado de humedad (Alcaraz, F., 2012). El sitiol “con grado de conservación menor” está cercano a la carretera, a viviendas y cultivos, así mismo, se evidenció presencia de tala (por lo menos 10 claros por esta causa) y tumba de árboles para la construcción de caminos peatonales a través del bosque, por estas razones este es un bosque con árboles de baja altura en su mayoría y un dosel no tan cerrado, asi como presenta una alta demanda de leña y madera; a diferencia del sitio2 “con grado de conservación mayor" que no se encuentra rodeado por ninguna de las anteriores, la única casa cercana al sitio se encuentra a aproximadamente 30 minutos caminando y aunque existe una trocha es delgada y poco transitada, además de no haberse encontrado presencia de tala en más de una ocasión, por tanto un bosque con una buena presencia de árboles altos y gruesos, así como un dosel bastante cerrado, dadas estas caracteristicas este sitio tiene un bosque de referencia adecuado para la comparación.
Con el fin de conocer el grado de alteración de los bosques donde se realizaron los muestreos y verificar las diferencias en el estado de conservación se llevó a cabo en cada sitio de muestreo una caracterización de la estructura de la vegetación, que como su nombre lo indica, se fundamenta en el estudio de la estructura o arquitectura comunitaria, que está definida por el ordenamiento en sentido vertical y horizontal de sus componentes. En sentido vertical, el atributo que mejor refleja el aspecto, es la estratificación mientras que en sentido horizontal, aparecen la densidad, el área basal y la cobertura (RangelCh, J.O \& Velásquez, A, 1997). El análisis de la distribución de clases de las alturas, de los valores del DAP y de las coberturas, facilita la comprensión de la dinámica de la vegetación y se pueden interpretar si los sitios muestreados estaban conservados o con cierto grado de alteración (Rangel-Ch, J.O \& Velásquez, A, 1997). De esta manera, los datos de campo que se utilizaron para la caracterización son: descripción general, Imagen satelital, densidad o número de individuos según la superficie de 
muestreo, altura, CAP (Cintura a la altura del pecho) y se siguieron las recomendaciones estipuladas por Rangel-Ch, J.O \& Velásquez, A, 1997.

Para conocer las especies del género Heliconius que se encuentran en las áreas de muestreo en los Municipios de Leticia y Puerto Nariño - Amazonas, se llevaron a cabo tres actividades, en primer lugar se hizo una revisión bibliográfica para conocer reportes de las especies de este género en estos sitios, posteriormente se realizaron visitas y revisión del género en las principales colecciones del país y finalmente se llevó a cabo una recolección permanente durante los meses de muestreo de las especies que iban apareciendo, los especímenes recolectados fueron montados y determinados en el Instituto de Ciencias Naturales de la Universidad Nacional de Colombia a partir de la Literatura, disección de genitalias y asesoría de los expertos. La recolección se hizo por 48 días de 9:00am - 4:00 pm para cada sitio de muestreo durante seis meses consecutivos, posteriormente se analizaron los datos de captura diaria por especie, y así poder analizar las diferencias entre la cantidad de individuos por especie en cada sitio. Para realizar las comparaciones se llevaron a cabo análisis de contraste de homogeneidad para determinar si los sitios si son diferentes significativamente en cuanto a la conservación de su bosque y de la diversidad y cantidad de individuos del género Heliconius.

\section{Resultados}

\section{Caracterización de la estructura de la vegetación}

Densidad de individuos. En la figura 2 se puede observar que el sitiol (con menor grado de conservación) presentó en todos los cuadrantes un menor número de individuos por metro cuadrado con respecto al sitio2; en general, en el primer sitio se contaron 842 individuos presentando así una densidad de 0,7 individuos por metro cuadrado. Para el sitio2 se presentó en todos los cuadrantes un mayor número de individuos por metro cuadrado de modo tal que presentó un total de 1.628 individuos y una densidad de 1,3 individuos por metro cuadrado, un 46,1\% más densidad que en el sitio1. Se llevó a cabo un contraste de homogeneidad por medio del Programa RWizard 1,1 (Guisande, C.\& et al., 2014). Se tiene en cuenta el resultado del G Test Williams, el cual dio 25,42 y un $\mathrm{p}<0,0001155$; por lo tanto, se rechaza la idea de que las muestras son homogéneas, es decir, existen diferencias significativas entre los dos sitios. Se puede decir que las diferencias entre los dos bosques se dan principalmente por el cuadrante N. 1, que en el caso del sitio1 es el más cercano a la carretera y a los sitios de vivienda y cultivos, lo que hace que hallan tan pocos individuos.

Distribución por categorías CAP. Para analizar la distribución de los individuos por categorías CAP, los resultados fueron agrupados en seis categorías: $1-10 \mathrm{~cm}, 11-$ $20 \mathrm{~cm}, 21-30 \mathrm{~cm}, 31-40 \mathrm{~cm}, 41-50 \mathrm{~cm}, 51-160 \mathrm{~cm}$. La tendencia general en los dos bosques es presentar mayor abundancia de individuos en la clase de menor diámetro, sus valores de abundancia decrecen hacia las clases de mayor CAP de tal manera que para el sitio N. 1 el $82 \%$ de los Individuos tienen un CAP menor de $10 \mathrm{~cm}$ y para el sitio $2 \mathrm{el}$ $78 \%$ de los individuos presentan este valor de CAP. Al igual que en el análisis de la densidad el sitio 2 presenta siempre un mayor número de individuos en todas las categorías, siendo más marcada la diferencia entre los dos sitios en la primera y dos últimas categorías donde la diferencia es muy ampliapara el sitio2. De esta manera, el número total de individuos con un CAP $\geq 10$ en todo el sitio1 fue de 154 y para el sitio2 de 345 (Figura 3). Los resultados de la prueba de contraste de homogeneidad muestran que los dos sitios son significativamente diferentes, el G Test Williams presenta un valor de 38,24 y un $\mathrm{p}<3.368 \mathrm{e}-07$, las diferencias están dadas principalmente por las dos últimas categorías, en los CAP de mayor amplitud, esto muy seguramente causado por la tala de árboles llevada a cabo en el sitio N. 1, donde muy pocos arboles alcanzan a completar CAP amplios.

Distribución por categorías de estratos arbóreos. No se diferencia un estrato arbóreo superior predominante en ninguno de los sitios (Figura 4), sin embargo, el sitio2 presenta un mayor número de individuos maduros con una altura mayor a 25 metros. El estrato arbustivo y subarboreo están muy bien representados en los dos sitios; en el sitio1 estos tienen una cobertura promedio del $88 \%$ y en el sitio 2 una cobertura promedio de $84 \%$, comparando con el estudio hecho por (Cantillo, E. \& Rangel-Ch, J.O, 2010) se puede establecer que los sitios en estudio pertenecen a la unidad de clasificación geomorfológica de Lomerío descrita por ellos, en donde encontraron que en promedio estas áreas tienen un $86 \%$ de estrato arbóreo inferior (Arbustivo) porcentaje muy similar al encontrado en este trabajo. Como se evidencio en el parámetro de CAP el sitio2 gana mayor diferencia en las últimas categorías y también en este caso el motivo de ausencia de individuos del estrato arbóreo superior en el

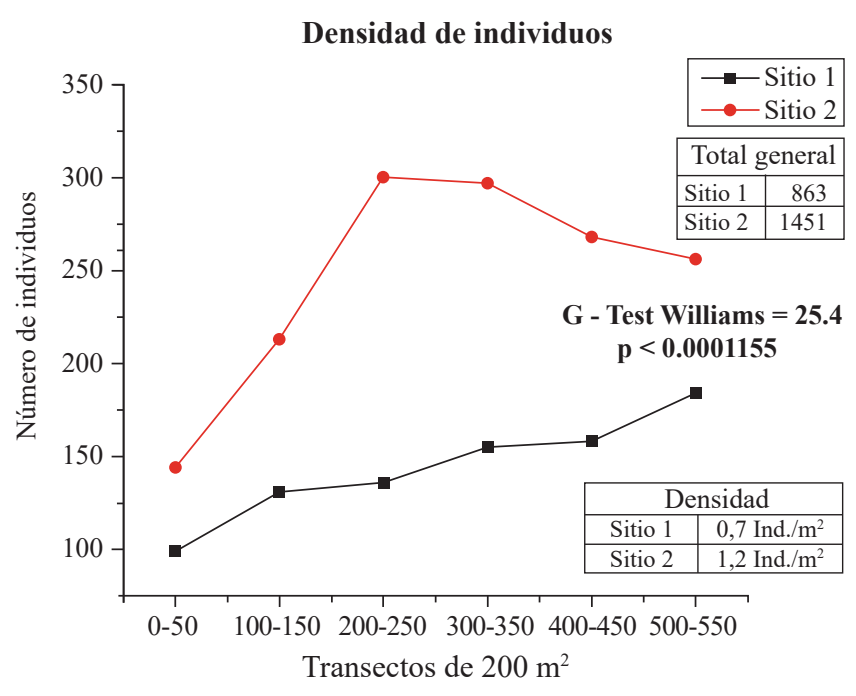

Figura 2. Gráfica de densidad de individuos - vegetación. 


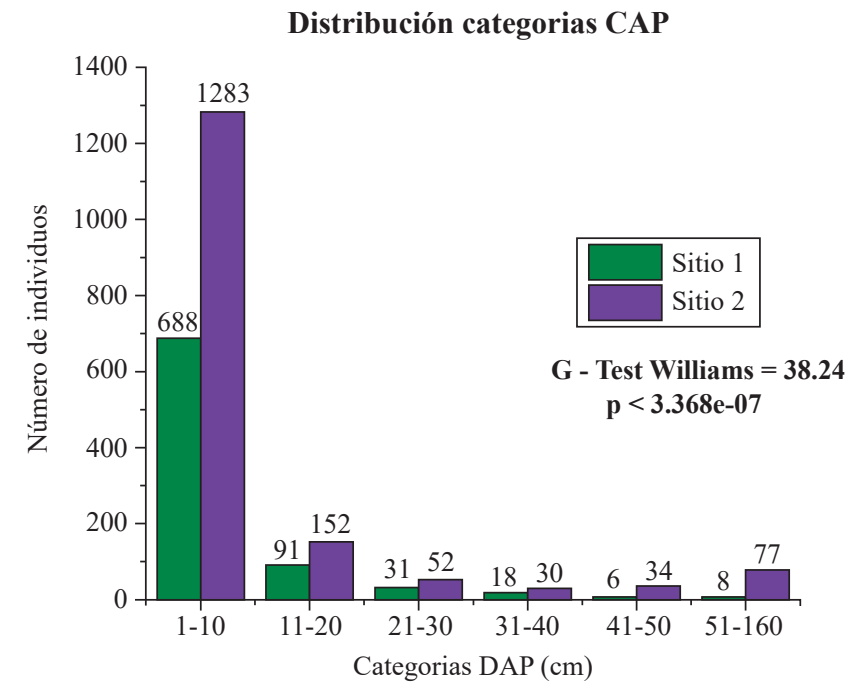

Figura 3. Gráfica de distribución categorías CAP - vegetación.

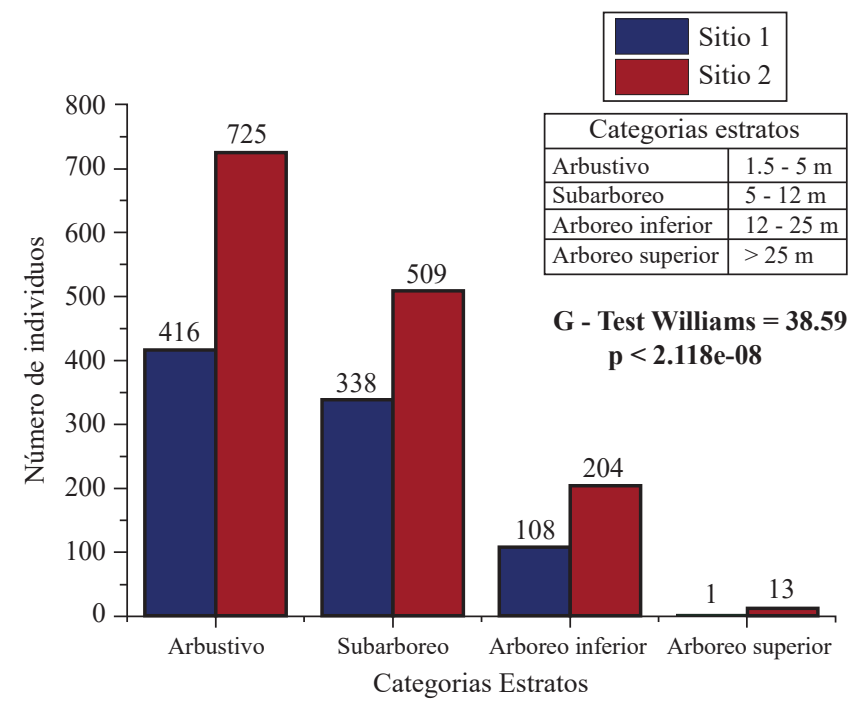

Figura 4. Gráfica de distribución en categorías de estratos vegetación.

sitio1 está relacionado con la afectación antrópica a la que es expuesto. Al igual que en las categorías anteriores los sitios son significativamente diferentes en cuanto a la altura de sus árboles (Test de G: p < 2.118e-08). Las categorías subarboreo y arbóreo superior son las que aportan más a la diferenciación de los sitios.

Comunidad de mariposas del género Heliconius de las áreas de estudio.

Reportadas en la literatura. Se llevó a cabo una búsqueda de información publicada sobre reportes de especies del género Heliconius encontradas en Colombia y particularmente en el Departamento del Amazonas, encontrando muy poca información publicada para este departamento, de modo tal, que este listado se hizo a partir de la revisión de los libros de mariposas "Butterflies of
America de D'Abrera Bernard, 1984 y “Heliconius and related genera. Lepidoptera: Nymphalidae. The genera Eueides, Neruda and Heliconius" de Holzinger, H. \& Holzinger, R., 1994, la página ilustrada de mariposas para América (http://butterfliesofamerica.com/ de (Warren, A. D, et al., 2013), un artículo sobre la lista de mariposas de Colombia "Biodiversidad de las mariposas (Lepidóptera: Rhopalocera) de Colombia" de Andrade, M.G., 2002 (Ver Anexo 1, http://www.raccefyn.co/index.php/raccefyn/article/ downloadSuppFile/382/1751).

Presentes en colecciones del país. La tabla 1 presenta las especies encontradas en las colecciones revisadas: Instituto de Ciencias Naturales -ICN-, Universidad Nacional de Colombia -UNAL-, Instituto Alexander von Humboldt IavH-, Universidad de los Andes, Colección Personal Jean F. Le Crom, Bogotá y Colección Universidad Nacional de Colombia - UNAL- Sede Medellín.

Recolectadas durante el periodo de muestreo en las áreas de estudio. La Figura 5 (sitio1) y la Figura 6 (sitio2) presentan las especies encontradas a lo largo de los seis meses de muestreo, en el sitio1 con menor grado de conservación se encontraron 9 especies y 14 subespecies y en sitio2 con Mayor grado de Conservación se encontraron 8 especies y 11 subespecies. Las especies que están encerradas en un cuadro rojo en el sitio1 son las especies que se recolectaron solo para ese sitio y las que están dentro de un cuadro azul son las que se recolectaron en una sola ocasión; asi mismo, las especies que están en cuadro rojo en el sitio2 son las que están ausentes para este sitio y las que están en azul nuevamente son las que se recolectaron en una sola ocasión.

La tabla 2 muestra el resumen de las especies encontradas, como es posible evidenciar en estos resultados se puede ver que hay especies y/o subespecies que no se encontraron en algunas de las tres actividades. Todas están presentes en la Literatura, no fue recolectada para los dos sitios la subespecie $H$. antiochus antiochus, la subespecie $H$. wallacei flavescens fue recolectada a $11 \mathrm{Km}$ del sitiol y en ninguna ocasión para el sitio2, las subespecies $H$. numata arcuella y $H$. hecale humboldti no fueron encontradas en el sitio2.

Para los dos sitios el número de días de muestreo fue de 48 y el promedio de individuos capturados por día para el sitiol es de 4.8 con una desviación estándar (SD) de 2.21 y para el sitio2 de 4.7 con una desviación estándar (SD) es de 2.17, con tres de días de $n=1(n=\#$ de capturas $)$ para el sitio 1 y cuatro días de $n=1$ para el sitio2, este sitio presenta una media mayor de 4.5 que en el sitio1 (4.0).

En la Figura 7, se observan los resultados del número de individuos capturados en cada uno de los sitios, en estas podemos observar como para el sitiol se realizaron un $22.7 \%$ más de capturas que en el sitio2 (Barra morada), aportada esta diferencia principalmente por las especies $H$. numata spp? y H. sara, las cuales si se encontraron en el sitio2 pero en una proporción considerablemente más baja. El resultado en cuanto a la especie $H$. numata concuerda 
Tabla 1. Especies y subespecies del género Heliconius presentes en las colecciones del país.

\begin{tabular}{|c|c|c|c|c|}
\hline Especie / Subespecie & $\begin{array}{c}\text { Instituto de Ciencias } \\
\text { Naturales - ICN- UNAL }\end{array}$ & $\begin{array}{l}\text { Universidad Nacional de } \\
\text { Colombia sede de Medellín }\end{array}$ & $\begin{array}{l}\text { Instituto Von } \\
\text { Humboldt }\end{array}$ & $\begin{array}{l}\text { Colección privada Jean } \\
\text { Francois Le Crom }\end{array}$ \\
\hline H. leucadia leucadia & $\mathrm{X}$ & & $\mathrm{X}$ & \\
\hline H. doris dives & $\mathrm{X}$ & & $\mathrm{X}$ & \\
\hline H. antiochus antiochus & $\mathrm{X}$ & & $\mathrm{X}$ & \\
\hline H. wallacei flavescens & $\mathrm{X}$ & $\mathrm{X}$ & & $\mathrm{X}$ \\
\hline H. sara sara & $\mathrm{X}$ & $X$ & $\mathrm{X}$ & \\
\hline H. erato reductimacula & $\mathrm{X}$ & & $\mathrm{X}$ & $\mathrm{X}$ \\
\hline H. erato lativitta & $\mathrm{X}$ & & $X$ & $X$ \\
\hline H. numata aurora & & $X$ & $\mathrm{X}$ & $\mathrm{X}$ \\
\hline H. numata silvana & $\mathrm{X}$ & $\mathrm{X}$ & $X$ & \\
\hline H. melpomene malleti & & $X$ & $X$ & $\mathrm{X}$ \\
\hline H. melpomene vicina & $\mathrm{X}$ & & $\mathrm{X}$ & $\mathrm{X}$ \\
\hline H. elevatus elevatus & $\mathrm{X}$ & & $X$ & $\mathrm{X}$ \\
\hline H. pardalinus butleri & $\mathrm{X}$ & & $X$ & $\mathrm{X}$ \\
\hline H. hecale humboldti & & & $\mathrm{X}$ & $\mathrm{X}$ \\
\hline
\end{tabular}

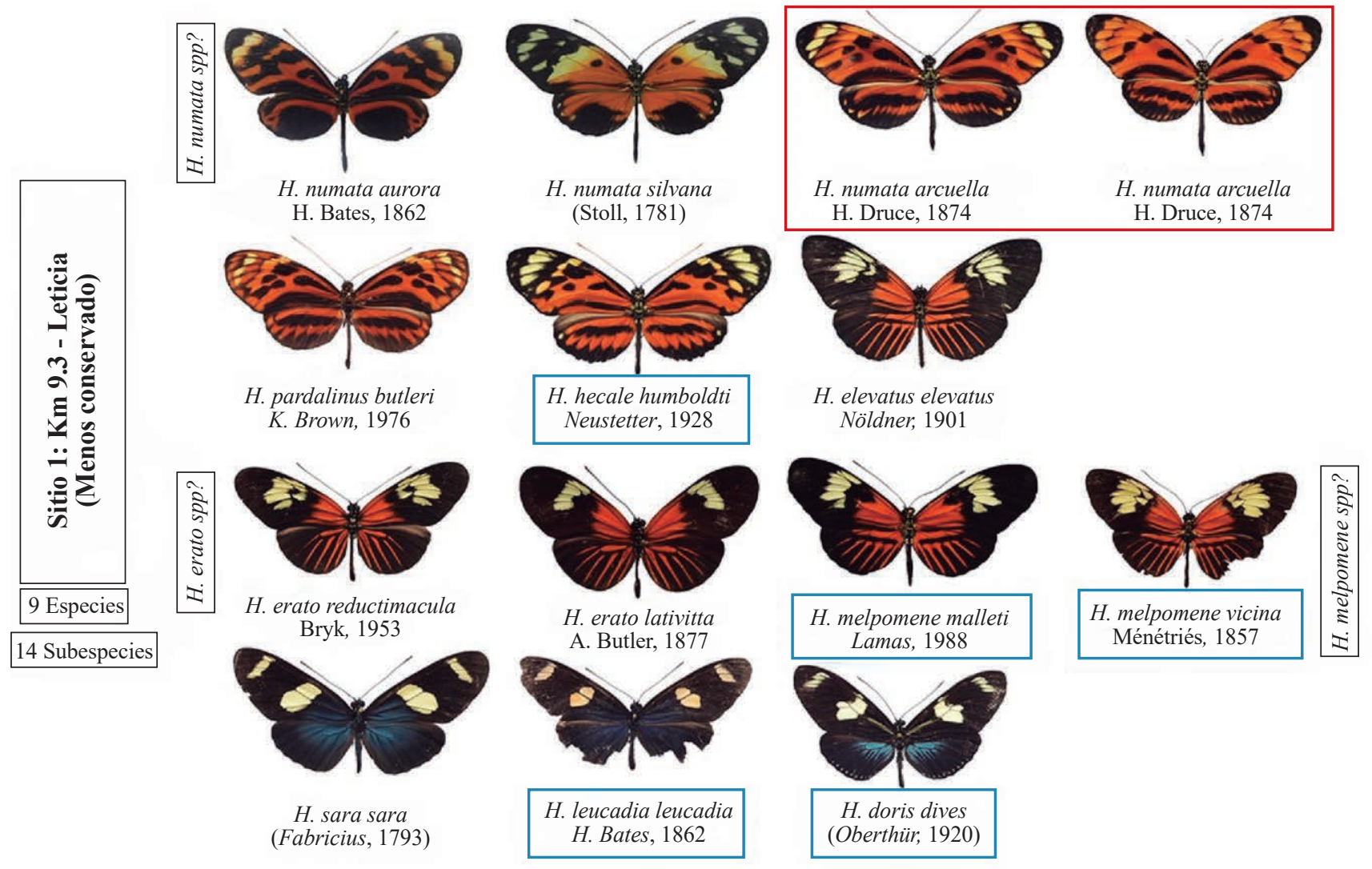

Figura 5. Especies y subespecies del género Heliconius recolectadas en el sitio 1, km 9.3 - Leticia.

con lo encontrado en otros estudios como por ejemplo en el de Joron, M., 2005, donde se afirma que esta especie se encuentra en una amplia diversidad de hábitats pero que son más comunes en Bosques Secundarios altos, como el del sitiol. Por otra parte, la especie H. pardalinus butleri no fue encontrada en el sitio 2 y si en el sitiol en muy baja cantidad y la especie $H$. melpomene fue encontrada en mayor cantidad en el sitio2 y una sola vez para el sitio1. En el análisis de contraste de Homogeneidad, se puede ver que el valor del G Test Williams es igual a 49.93; $\mathrm{p}<1.434 \mathrm{e}-09$, por lo que se puede decir que en cuanto al número de marcados los dos sitios son significativamente diferentes y además la gráfica nos muestra que esta diferencia es aportada principalmente por las especies $H$. melpomene spp? y $H$. sara. 


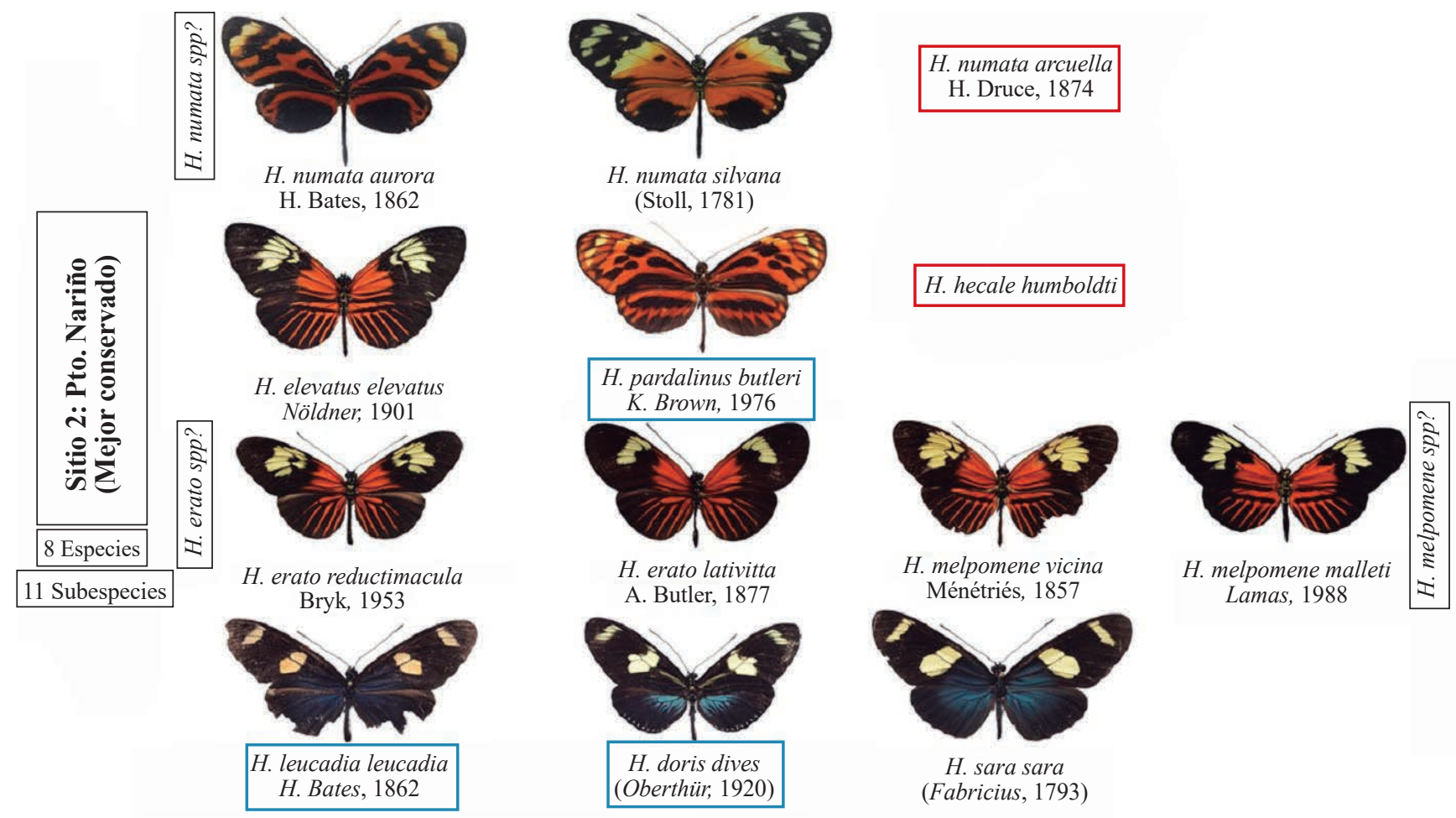

Figura 6. Especies y subespecies del género Heliconius recolectadas en el sitio 2 Puerto Nariño.

Tabla 2. Resumen de las especies del género Heliconius en el Amazonas Colombiano.

\begin{tabular}{lcccc}
\hline Taxa & Literatura & Colecciones & \multicolumn{2}{c}{ Recolectada } \\
\cline { 3 - 5 } & & & Sitio 1 & Sitio2 \\
\hline H. leucadia leucadia & $\mathrm{X}$ & $\mathrm{X}$ & $\mathrm{X}$ & $\mathrm{X}$ \\
\hline H. doris dives & $\mathrm{X}$ & $\mathrm{X}$ & $\mathrm{X}$ & $\mathrm{X}$ \\
H. antiochus antiochus & $\mathrm{X}$ & $\mathrm{X}$ & - & - \\
\hline H. wallacei flavescens & $\mathrm{X}$ & $\mathrm{X}$ & $\mathrm{X} *$ & - \\
H. sara sara & $\mathrm{X}$ & $\mathrm{X}$ & $\mathrm{X}$ & $\mathrm{X}$ \\
H. erato reductimacula & $\mathrm{X}$ & $\mathrm{X}$ & $\mathrm{X}$ & $\mathrm{X}$ \\
H. erato lativitta & $\mathrm{X}$ & $\mathrm{X}$ & $\mathrm{X}$ & $\mathrm{X}$ \\
\hline H. numata aurora & $\mathrm{X}$ & $\mathrm{X}$ & $\mathrm{X}$ & $\mathrm{X}$ \\
H. numata silvana & $\mathrm{X}$ & $\mathrm{X}$ & $\mathrm{X}$ & $\mathrm{X}$ \\
\hline H. numata arcuella & $\mathrm{X}$ & & $\mathrm{X}$ & - \\
H. numata arcuella & $\mathrm{X}$ & & $\mathrm{X}$ & - \\
\hline H. melpomene malleti & $\mathrm{X}$ & $\mathrm{X}$ & $\mathrm{X}$ & $\mathrm{X}$ \\
H. melpomene vicina & $\mathrm{X}$ & $\mathrm{X}$ & $\mathrm{X}$ & $\mathrm{X}$ \\
\hline H. elevatus elevatus & $\mathrm{X}$ & $\mathrm{X}$ & $\mathrm{X}$ & $\mathrm{X}$ \\
H. pardalinus butleri & $\mathrm{X}$ & $\mathrm{X}$ & $\mathrm{X}$ & $\mathrm{X}$ \\
\hline H. hecale humboldti & $\mathrm{X}$ & $\mathrm{X}$ & $\mathrm{X}$ & - \\
\hline
\end{tabular}

*A $11 \mathrm{Km}$ del sitio de muestreo en dirección hacia el Rio Amazonas.

Durante el proceso de muestreo se notó que era muy difícil la captura de los individuos debido a que estas presentan en una buena proporción del día una altura de vuelo mayor a 6 metros y solo bajan a los claros del bosque en los momentos del día en el que la temperatura asciende considerablemente; por esta razón se decidió que era pertinente realizar un conteo visual que permitiera tener una mejor idea de la comunidad de mariposas de este género. De esta manera, en la Figura 8 se muestran dos gráficas de la cantidad de observaciones en total y por especie en cada uno de los sitios. Es posible evidenciar, que para el sitio2 (Derecha) se hicieron más del doble de observaciones en el total general y esta diferencia se da principalmente por una cantidad mucho mayor de individuos de las especies $H$. numata spp? y H. erato spp?, así mismo como en los resultados de capturas las especies $H$. sara y $H$. pardalinus no fueron observadas en el sitio2. El contraste de homogeneidad del número de observaciones nos confirma entonces que los dos sitios son significativamente diferentes en cuanto al número de individuos observados, este análisis muestra un valor en el G Test Williams igual a 67,03; $\mathrm{p}=1.843 \mathrm{e}-14$. Del mismo modo, las especies que aportan principalmente a esta diferencia son $H$. sara y $H$. pardalinus con un mayor número de observaciones para el sitiol y $H$. erato spp? para el sitio2.

Se obtuvieron además de las especies y subespecies de Heliconius antes descritas un número de mariposas y polillas que presentan una morfología similar a las mismas, las cuales son llamadas especies miméticas, estas se mencionan a continuación ya que este conocimiento ha sido de gran importancia desde el tiempo de Darwin y Bates y permitió el desarrollo del concepto original de mimetismo, en el cual estas mariposas juegan un rol fundamental como modelos no palatables y ellos han permanecido como centro en el 

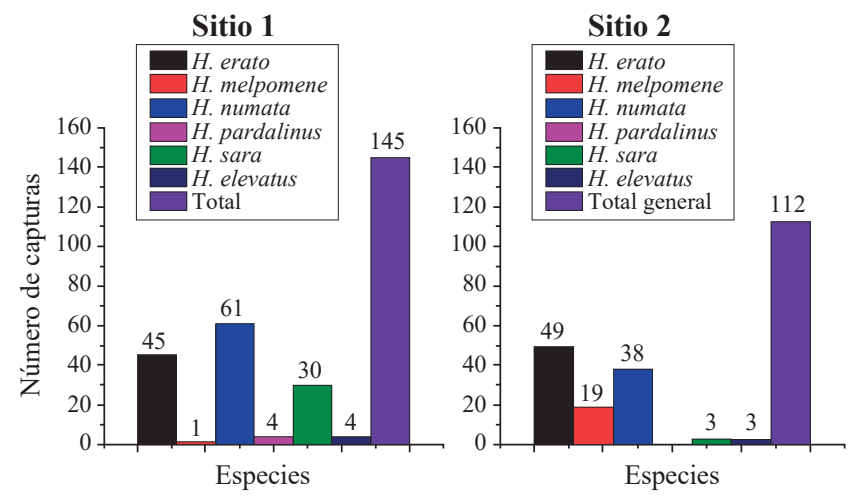

Figura 7. Número de marcajes por especie en el sitio 1 (menor estado de conservación) y en el sitio 2 (mayor estado de conservación).
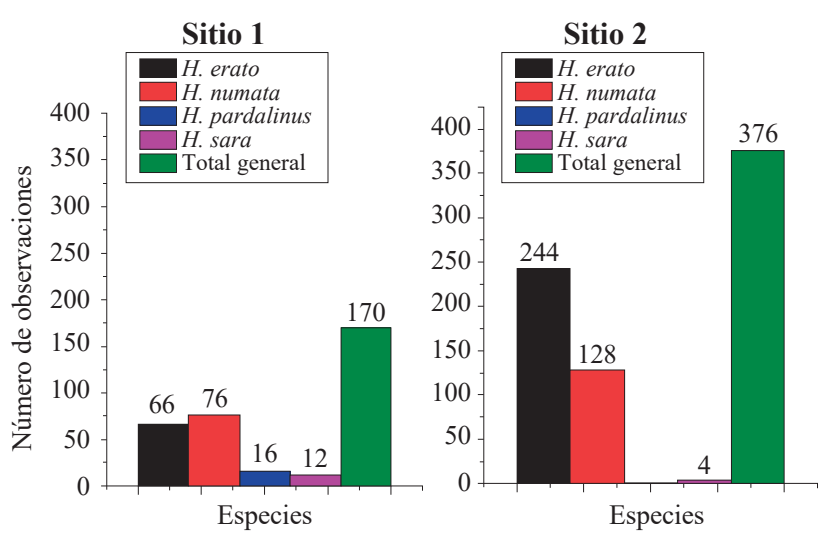

Figura 8. Número de observaciones por especie en el sitio 1 (menor estado de conservación) y en el sitio 2 (mayor estado de conservación).

desarrollo de la teoría y la evolución de la no palatabilidad (Poulton, 1887; Itringham, 1916; Fisher, 1930; Turner, 1965, 1970, 1976, 1983; Benson, 1971; Brown, Sheppard \& Turner, 1974; Sheppard, et al., 1985; Chai, 1990; Mallet \& Singer, 1987; Mallet, 1993; Mallet \& Gilbert, 1995; Brower, 1996. En: Penz, C., 1999), es así como este conocimiento se hace pertinente ya que evidencia la importancia de la conservación de este grupo como individuos involucrados en una variedad de procesos no solo ecológicos sino también evolutivos. En el Anexo 2, http://www.raccefyn.co/index.php/raccefyn/article/download SuppFile/382/1752, se muestran las especies miméticas del género Heliconius en las áreas de muestreo, se identificaron especies de la subfamilia Ithomiinae, del género Neruda de la subfamilia Heliconiinae y dos especies de polillas de la Subfamilia Arctiinae; la especie que más miméticos presenta es la especie $H$. numata, esta no solo presenta apariencias fenotípicas muy diferentes en el mismo sitio sino que también presenta convergencia mimética local casi perfecta con otras especies, cada uno de los diferentes morfos es un preciso mimético de una especie diferente del Género Melinaea (Joron Mathieu, et al., 2006), en la figura se muestran dos de los pares miméticos de las tres formas de $H$. numata encontradas, así como también, se muestra la especie de polilla Chetone histrio Boisduval, 1870, que también la mimetiza; posteriormente, se muestra la especie mimética de $H$. pardalinus que pertenece al mimo género Melinaea; la siguiente especie dentro de Heliconius que presenta varios miméticos es $H$. erato, en primer lugar está la especie Neruda aeode y luego la especie de polilla Chetone phyleis (Druce, 1885).

\section{Discusión}

A partir del estudio de la estructura de la vegetación de los dos bosques en estudio, se confirmo por medio de los análisis de contraste de homogeneidad que los dos sitios son significativamente diferentes en el grado de conservación de los bosques, que el sitio 2 presenta un grado de conservación mayor y que la degradación a la que está siendo sujeto el sitiol ha alterado la disponibilidad de las fuentes de alimentos de néctar y de las plantas hospederas de estas especies de mariposas, por un lado, ha favorecido el crecimiento de las pasifloras en el borde del bosque, ya que las semillas de estas plantas pueden germinar solo en áreas abiertas donde el suelo está expuesto a la luz (Gilbert, L., 1982), y por otro lado, la cercanía a las casas ha hecho que haya una mayor oferta de polen fuera del bosque, lo que justifica en gran parte que la mayoría de la captura de individuos haya sido en el borde del sitio teniendo en cuenta que el recurso de polen en Heliconius parece ejercer más grande influencia en los patrones de movimiento de los adultos que incluso lo que hace el recurso larval (Ehrlich \& Gilbert, 1973. En: Gilbert, L., 1984).

En cuanto a la diversidad de especies del género Heliconius en los dos sitios, la diferencia fue a favor del sitiol ya que en este sitio se capturó una especie más (Heliconius hecale) y dos subespecies más de H. numata; en el primer caso, es importante, tener en cuenta que subespecie H. hecale humboldti si ha sido reportada para el sitio2 en la colección de J.F. Le crom en el año 1998 en un mes diferente al de los muestreos (julio), por lo que si debe estar allí y su ausencia deberse a la temporada climática en la que se hizo el muestreo.

La ausencia de las subespecies $H$. numata arcuella en el sitio 2 puede deberse a que esta especie es comúnmente habitante de bosques secundarios (Joron Mathieu, et al., 2006) como el bosque del sitiol y a que esta especie es generalista en el uso de su planta hospedera, ya que se le vio ovipositar en cuatro pasifloras (P. edulis, P. cuadrangulares, $P$. vitifolia y $P$. ligularis), así mismo, estas pasifloras germinan y crecen solamente en áreas con disturbio tales como en árboles caídos, derrumbes y bancos de semillas (Mallet, J., 1986) por lo que es mayor la densidad de este recurso en comunidades simples como en el sitiol que en agregaciones vegetales más complejas como el sitio2. Esta subespecie es registrada por primera vez en el país y confirma el 
polimorfismo intraespecífico que este grupo presenta (Bates, 1862; Eltringham, 1916; Turner, 1976; Brown, 1979. En: Brower, A., 2011).

Por otra parte, en el caso de la subespecie de $H$. antiochus antiochus, la cual no fue recolectado en los dos sitios, es reportada en la colección del ICN- UNAL para las comunidades de Nazareth, Arara y Macedonia y en la colección del IAvH para Leticia. Lo que permite inferir que la subespecie debe estar para los dos sitios y en la misma temporada climática, dado que las fechas de recolecta coinciden con la fecha de muestreo, de tal manera, que la razón por la que no se capturo en ninguno de los dos sitios debe obedecer más a la baja probabilidad de captura que puede ser por un comportamiento de vuelo restringido al dosel del bosque o a áreas abiertas, así como también puede deberser a un número bajo de individuos en sus poblaciones. Esto mismo sucede para la subespecie $H$. wallacei flavescens, la cual fue reportada en la Colección del ICN-UNAL para la vereda de Nazareth y en el Hotel On vacation cerca a la Ciudad de Leticia, en la colección de la UNAL- Sede Medellin para Leticia y en la colección de J.F. Le crom para el Municipio de Puerto Nariño, por lo tanto, se puede inferir que esta debe estar en los dos sitios de muestreo y en la misma temporada climática.

En cuanto al número de individuos capturados se muestra que los sitios son significativamente diferentes y que el sitiol en general presenta un $22.7 \%$ más de capturas que el sitio2, sin embargo, es importante señalar que esto nos necesariamente indica que haya mayor cantidad de individuos en el sitiol que en el sitio2 sino que es el resultado del efecto de muestreo, ya que, el bosque de este último sitio al ser más cerrado y más alto no permitió la captura de más cantidad de individuos, esto es corroborado con los resultados del conteo visual, el cuál fue mucho más alto en el sitio2.

Se hace necesario mencionar que en cuanto a las capturas, hubo especies que fueron capturadas en una sola ocasión durante los siete meses de muestreo en las mismas áreas de estudio o por fuera de ellas como $H$. antiochus antiochus, $H$. wallacei flavescens, H. doris dives, H. hecale humboldti y H. leucadia leucadia; muestreos anteriores a este, en otras épocas y otros sitios han permitido ver que en general estas especies se ven de manera muy poco frecuente; con respecto a esto, Pollard y Yates, 1993 (En: Gaskin, D., 1995) notaron que algunas especies pueden ser subcontadas en muestreos de transectos, esto debido a que algunas especies pueden presentar concentraciones en colonias locales y estás son perdidas en el muestreo, o a que las especies estén grandemente restringidas al dosel y fuera de vista de los observadores, o que sean de baja visibilidad por su coloración criptica, o que tengan un comportamiento o vuelo en márgenes sombreadas o a que realmente sean raras como una función propia de poblaciones pequeñas. El inconveniente es que determinar si un taxón es verdaderamente raro puede ser difícil y solo los estudios de campo a largo término son la única manera de resolver muchos de estos enigmas (Gaskin, D., 1995). En el caso de que una especie presente características de una especie rara no se debe asumir inmediatamente que es un efecto de actividades antropogénicas, ya que algunas poblaciones pueden sostenerse en bajas densidades por limitación de recursos, predadores, parásitos o enfermedades; sin embargo, la investigación debe ser de alta prioridad ya que cuando las distribuciones son claramente localizadas y las densidades poblacionales bajas no son balanceadas por alta movilidad además de la especieser "rara" puede también ser "vulnerable" (Gaskin, D., 1995). De modo tal, que es importante priorizar nuevas investigaciones sobre estas especies que permitan conocer el motivo de su baja captura.

Finalmente, no es sorpresa del todo que la diferencia en cuanto a la diversidad de las especies de este género entre los dos sitios haya favorecido al sitio1, ya que como han mencionado otros autores, como Horner, M., Daily, G., Ehrlich, P., Boggs, C., (2003), se ha encontrado que los bordes entre bosques y áreas abiertas algunas veces tienen riquezas de especies relativamente altas, esto posiblemente dado por la presencia de viviendas y áreas de agricultura cercanas a los bosques que tienen una alta importancia en recursos como las plantas hospederas, frutas en descomposición, flores con néctar y polen para las mariposas, por lo que podría también tener un efecto indirecto en la diversidad de las mariposas alrededor del bosque, este fenómeno es conocido como efecto de borde. Por otro lado, Ehrlich, P., (1984) concluye que uno de los factores que más influencian la estructura de una población es la distribución y abundancia de los recursos nutricionales, los cuales dependen completamente del grado de conservación del bosque en general, y en el caso particular de los Heliconius observados en este trabajo se comprueba que los individuos se mueven entre los recursos de las larvas y de los adultos, los cuales están separados, siendo estos factores determinantes en su desplazamiento.

Así es importante notar que la afectación de los problemas ambientales actuales sobre las poblaciones de mariposas depende en gran parte del nivel de los mismos, dado que se ha visto que a niveles intermedios (Fragmentación) puede introducir recursos favorables para las poblaciones; así mismo, es importante tener en cuenta que la afectación es diferencial con respecto al grupo de mariposas en particular, en el caso de Heliconius, la plasticidad ecológica que presenta le permite a varias de sus especies (H. numata) responder favorablemente al disturbio intermedio, como el caso de la especie $H$. numata, la cual en el sitiol se vio favorecida, presentando subespecies diferentes y mayor cantidad de individuos, esto puede deberse a que esta especie es comúnmente habitante de bosques secundarios (Joron Mathieu, et al., 2006) y/o al favorecimiento también de los recursos propios de esta especie, como su planta hospedera, lo que ha sido generado por la alteración a la dinámica natural del bosque. Esto también podría deberse a que esta especie presenta la habilidad de usar un gran rango de recursos de adultos y de larvas, cambiando de 
comportamiento y preferencias de acuerdo al ambiente, lo que es conocido como plasticidad ecológica, esto visto para H. erato phyllis por Ramos, R. \& Freitas, A., 1999 y contrario a lo manifestado por estos autores para $H$. numata spp?, los cuales encontraron que en su áreas de estudio esta especie estaría restringida a sectores limitados de hábitats de bosque.

\section{Conclusiones}

Si bien los sitios son significativamente diferentes en su estado de conservación, se puede decir que el sitiol presenta una afectación del bosque que se considera en un grado no tan alto como podría llegar a ser en el futuro, por tanto, se considera que los resultados de este trabajo en cuanto a un mayor número de individuos en el sitio más conservado, es una primera muestra de la afectación del grado de conservación en las poblaciones de mariposas y que el resultado de una diversidad mayor en el sitiol, se puede explicar en parte por la dificultad de captura en el sitio2 y por otra parte se debe considerar que este género en particular se le ha visto en otros estudios ser más resistentes al disturbio gracias a la plasticidad ecológica que muchas de sus especies muestran, por tanto, es importante no hacer generalizaciones al resto de especies de las mariposas ni en todos los sitios, y llevar a cabo más investigaciones que permitan resolver preguntas como: ¿Con el avance de la alteración de los bosques se espera que también avancen las alteraciones en las poblaciones de mariposas? ¿Si esto sucede en este grupo de mariposas que podría estar sucediendo con otros grupos? ¿Podrían algunos de estos grupos ser más vulnerables a la disminución de su habitat? ¿Qué está pasando con especies como $H$. leucadia leucadia que en siete meses solo fue capturada en tres ocasiones para los dos sitios? ¿Es pertinente esperar a que las poblaciones de mariposas se vean realmente amenazadas para intervenir como en muchas otras ocasiones se ha hecho con otros seres vivos? ¿Qué acciones o medidas se pueden adelantar que permitan la conservación de los bosques del Amazonas colombiano y por ende de los seres vivos que habitan en ellos?

Finalmente, se considera que el Amazonas es un área de suma importancia para la conservación, no solo por los servicios ambientales que presta sino que también tiene un valor intrínseco al ser un ecosistema que alberga una gran variedad de formas de vida, como el género Heliconius, al ser una zona de contacto con presencia de una gran variedad de polimorfos locales con hibridación intraespecífica, de tal manera que es un banco genético, con gran cantidad de datos ecológicos y evolutivos y que el país debe poner mayor interés y recursos económicos a las estrategias ya establecidas y debe gestionar medidas de uso y conservación como: reducir la pérdida de hábitat, fomentar programas de investigación, fomentar las instituciones tanto públicas como privadas envueltas en la conservación y manejo de los bosques y la biodiversidad aumentar el control y la protección de los seres vivos ante el comercio y la explotación ilegal.

\section{Información suplementaria}

Anexo 1. Especies del género Heliconius reportados en la literatura. Vea el anexo 1 en: http://www.raccefyn.co/index.php/raccefyn/ article/downloadSuppFile/382/1751

Anexo 2. Especies miméticas del género Heliconius halladas en los sitios de muestreo en el Amazonas Colombiano. Vea el anexo 2 en: http://www.raccefyn.co/index.php/raccefyn/article/download SuppFile/382/1752

\section{Agradecimientos}

A la Universidad Nacional de Colombia y al Instituto de Ciencias Naturales por los espacios y tiempos brindados, a la Fundación Omacha por el apoyo logístico en el Municipio de Puerto Nariño, a la Empresa Mariposas del Amazonas por su apoyo logístico en el Municipio de Leticia y a la Secretaria de Educación del Distrito de Bogotá por la Comisión de Estudios brindada a la estudiante. Al Profesor André Lucci Freitas por sus aportes y enseñanzas.

\section{Conflicto de intereses}

Los autores declaran que no tienen conflicto de intereses.

\section{Referencias}

Alcaraz, F. (2012). Geobotánica, Tema 22. Selvas ecuatoriales y tropicales. Universidad de Murcia, España. Recuperado de: http://www.um.es/docencia/geobotanica/ficheros/tema22.pdf

Andrade, M.G. (2002). Biodiversidad de las mariposas (Lepidóptera: Rhopalocera) de Colombia. Monografias Tercer Milenio. 2: 153-172.

Andrade-C., M.G. (1998). Utilización de las Mariposas como bioindicadores del tipo de hábitat y su biodiversidad en Colombia. Revista de la Academia de las Ciencias Exactas, Fisicas y Naturales. 22 (84): 407-421.

Araujo, A.M. (1980). Estudos genéticos e ecológicos em Heliconius erato (Lepidoptera, Nymphalidae). Actas IV Congr. Latinoam. Genetica. 2: 199-206.

Brower, A. (2011). Hybrid speciation in Heliconius butterflies? A review and critique of the evidence. Genetica. 139: 589609. http://doi.org/10.1007/s10709-010-9530-4

Brown, K. (1981). The biology of Heliconius and related genera. Annual review entomology. 26: 427-456.

Cantillo, E., \& Rangel-Ch, J.O. (2010). La estructura y la riqueza en los bosques del Amazonas colombiano. En: Colombia Diversidad Biótica VII (Vol. VII). Universidad Nacional de Colombia, Instituto de Ciencias Naturales, Instituto de hidrología, Meteorología y estudios Ambientales (IDEAM)Ministerio del Medio Ambiente, Comité de Investigaciones y Desarrollo Científico-CINDEC.U.N, Academia Colombiana de Ciencias Exactas, Físicas y Naturales. Bogotá D.C.

Cook, L., Thomason, E., \& Young, A. (1976). Population structure, dynamics and dispersal of the tropical butterfly Heliconius charitonius. Journal of animal ecology. 45 (3): 851-863. 
D'Abrera Bernard. (1984). Butterflies of the Neotropical Region. Parte II: Danaidae, Ithomiidae, Heliconidae \& Morphidae. (Vol. 2). Hill House.

Ehrlich, P. (1984). The structure and dynamics of butterflies populations. En The biology of butterflies (pp. 25-40). Florida: The Royal Entomological Society. Academic press.

Ehrlich, P., \& Gilbert, L. (1973). Population structure and dynamics of the Tropical Butterfly Heliconius ethilla. Biotropica, 5 (2): 69-82.

Gaskin, D. (1995). Butterfly conservation programs must be based appropiate ecological information. Proceedings of the entomological society of Ontario, 126: 15-27.

Gilbert, L. (1982). The Coevolution of a Butterfly and a Vine, Scientific American, INC, 247 (8): 110-121.

Gilbert, L. (1984). The biology of butterflies communities. En The biology of butterflies (pp. 41-54). Florida: The Royal Entomological Society. Academic press.

Guisande, C., \& et al. (2014). RWizard Software. Universidad de Vigo. España. Recuperado a partir de http://www.ipez.es/ RWizard.

Holzinger, H., \& Holzinger, R. (1994). Heliconius and related genera. Lepidoptera: Nymphalidae. The genera Eueides, Neruda and Heliconius. (Sciences Nat ed.). Francia: Sciences Nat.

Horner, M., Daily, G., Ehrlich, P., Boggs, C. (2003). Countryside Biogeography of Tropical Butterflies. Conservation Biology, 17 (1): 168-177.

IUCN. (2016). IUCN Red List. Recuperado a partir de http://www. iucnredlist.org/

Jiggins, C.D., M. Linares, R. E. Naisbit, C. Salazar, Z. H. Yang, \& J. Mallet. (2001). Sex-linked hybrid sterility in a butterfly. Evolution, 55: 1631-1638.

Joron, M. (2005). Polymorphic mimicry, microhabitat use, and sex-specific behaviour. Journal of evolutionary Biology. 18: 547-556. http://doi.org/10.1111/j.1420-9101.2005.00880.x

Joron Mathieu, Papa Riccardo, Beltran Margarita, Chamberlain Nicola, Maravéz Jesus, Baxter Simon, ... Jiggins C. (2006). A Conserved Supergene Locus Controls Colour Pattern Diversity in Heliconius Butterflies. PLoS Biology, 4 (10): 1831-1840. http://doi.org/10.1371/journal.pbio.0040303
Mallet, J. (1986). Dispersal and gene flow in a butterfly with home range behaviour: Heliconius erato (Lepidoptera: Nymphalidae), Oecología. 68: 210-217.

Mallet, J., \& L. E. Gilbert. (1995). Why are there so many mimicry rings? Correlations between habitat, behaviour and mimicry in Heliconius butterflies. Biological Journal of the Linnean Society, 55: 159-180.

Mallet, J.L.B., \& D.A. Jackson. (1980). The ecology and social behavior of the Neotropical butterfly Heliconius xanthocles Bates in Colombia. Zoological Journal of the Linnean Society, 70: 1-13.

Penz, C. (1999). Higher level phylogeny for the passion-vine butterflies (Nymphalidae, Heliconiinae) based on early stage and adult morphology. Zoological Journal of the Linnean Society, 127: 277-344. http://doi.org/1998.0187

Quintero, H. E. (1988). Population dynamics of the butterfly Heliconius charitonius L. in Puerto Rico. Caribbean Journal of Science, 24: 155-160.

Ramos, R., \& Freitas, A. (1999). Population biology and wing color variation in Heliconius erato phyllis (Nymphalidae). Journal of the Lepidopterists 'Society, 53 (1): 11-21.

Rangel-Ch, J.O, \& Velásquez, A. (1997). Metodos de estudio de la Vegetación. En Colombia. Diversidad Biotica II. Tipos de vegetación en Colombia. (Vol. II, p. 436). Bogotá D.C.: Universidad Nacional de Colombia, Instituto de Ciencias Naturales, Instituto de hidrología, Meteorología y estudios Ambientales (IDEAM)-Ministerio del Medio Ambiente, Comité de Investigaciones y Desarrollo Científico-CINDEC. U.N, Academia Colombiana de Ciencias Exactas, Físicas y Naturales.

Rosser, N., Phillimore, A., Huertas, B., Willmott, K., \& Mallet, J. (2012). Testing historical explanations for gradients in species richness in heliconiine butterflies of tropical America. Biological Journal of the Linnean Society. 105: 479-497.

Warren, A. D, K. J. Davis, E. M. Stangeland, J. P. Pelham, \& N. V. Grishin. (2013). Illustrated Lists of American Butterflies. Recuperado a partir de http://www.butterfliesofamerica.com 